\title{
Palladium-catalyzed amination of aryl sulfides with anilines.
}

\section{$\operatorname{AUTHOR}(\mathrm{S})$ :}

Sugahara, Tomohiro; Murakami, Kei; Yorimitsu, Hideki; Osuka, Atsuhiro

\section{CITATION:}

Sugahara, Tomohiro ...[et al]. Palladium-catalyzed amination of aryl sulfides with anilines.. Angewandte Chemie - International Edition 2014, 53(35): 9329-9333

\section{ISSUE DATE:}

2014-07-07

URL:

http://hdl.handle.net/2433/198446

\section{RIGHT:}

This is the peer reviewed version of the following article: Sugahara. T., Murakami, K. Yorimitsu, H. and Osuka, A. (2014), Palladium-Catalyzed Amination of Aryl Sulfides with Anilines. Angew. Chem. Int. Ed., 53: 9329-9333, which has been published in final form at http://dx.doi.org/10.1002/anie.201404355.; 許諾条件により本文ファイルは2015-07-07に公開 ; この論文は出版社版でありません。引用の際には出版社版をご確認ご利用ください。; This is not the published version. Please cite only the published version. 
Synthetic Method

\title{
Palladium-Catalyzed Amination of Aryl Sulfides with Anilines**
}

\author{
Tomohiro Sugahara, Kei Murakami, ${ }^{*}$ Hideki Yorimitsu, ${ }^{*}$ and Atsuhiro Osuka
}

\begin{abstract}
A combination of a palladium-NHC catalyst and potassium hexamethyldisilazide effects amination of aryl sulfides with anilines to afford $a$ wide variety of diarylamines. The reaction conditions are versatile enough to convert even bulky orthosubstituted aryl sulfides. This amination is thus applicable to new modular synthesis of $\mathrm{N}$-arylcarbazoles from orthobromothioanisoles. Since aryl sulfoxides undergo extended Pummerer reactions to afford ortho-substituted aryl sulfides, the Pummerer products are hence useful substrates for the amination to culminate in efficient syntheses of 2-anilinobenzothiophene and indole as proof-of-principle of the utility of extended Pummerer reaction/amination cascade.
\end{abstract}

$\boldsymbol{T}_{\text {ransition-metal-catalyzed amination of aryl halides }}{ }^{[1,2]}$ has been attracting considerable attentions from material and medicinal chemists because a significant number of functional molecules ${ }^{[3]}$ and drugs ${ }^{[4]}$ contain arylamine structures. Although aminations of inert C-O bonds of phenol derivatives such as mesylate, ${ }^{[5]}$ tosylates, ${ }^{[6]}$ carbamates, ${ }^{[7]}$ sulfamates, ${ }^{[7 a, 8]}$ pivalate esters, ${ }^{[9]}$ phosphate, ${ }^{[10]}$ and methyl ethers ${ }^{[11]}$ have been reported, it is still difficult to convert CS bonds of aryl sulfides into C-N bonds. ${ }^{[12,13]}$ The difficulty would originate from reluctant transmetalation due to strong interaction between a cationic transition metal and an anionic thiolate.

Our recent studies showed that palladium and nickel catalysts having an $\mathrm{N}$-heterocyclic carbene (NHC) ligand are sufficiently active for $\mathrm{C}-\mathrm{C}$ bond forming cross-coupling reactions of aryl sulfides. ${ }^{[14]}$ We envisioned that such electron-donating and bulky ligands would also help catalytic amination of aryl sulfides. This

[*] T. Sugahara, Prof. Dr. K. Murakami, Prof. Dr. H. Yorimitsu, Prof. Dr. A. Osuka

Department of Chemistry, Graduate School of Science, Kyoto University

Kitashirakawa, Sakyo-ku, Kyoto 606-8502 (Japan)

Fax: (+81) 75-753-3970

E-mail: yori@kuchem.kyoto-u.ac.jp

Prof. Dr. K. Murakam

The Hakubi Center for Advanced Research

Kyoto University

E-mail:kmura@kuchem.kyoto-u.ac.jp

Prof. Dr. H. Yorimitsu

ACT-C, Japan Science and Technology Agency.

[**] This work was supported by Grants-in-Aid from MEXT (Nos. 24106721 "Reaction Integration" and 25107002 "Science of Atomic Layers") and from JSPS (Nos.: 25220802 (Scientific Research (S)), 24685007 (Young Scientists (A)), 23655037 and 26620081 (Exploratory Research)). K.M. acknowledges JSPS Postdoctoral Fellowship for Young Scientists.

Supporting information for this article is available on the WWW under http://dx.doi.org/10.1002/anie.201xxxxxx.((Please delete if not appropriate)) was indeed the case and here we report amination reactions of aryl sulfides with aryl amines using a $\mathrm{Pd} / \mathrm{NHC}$ catalyst.

First, we optimized reaction conditions for the amination (Table 1). Treatment of thioanisole with $p$-toluidine (1.5 equiv) in the presence of Pd-PEPPSI-IPr ${ }^{[1 \mathrm{f}]}$ (5 mol\%) and $\mathrm{K}_{2} \mathrm{CO}_{3}$ (3 equiv) in dioxane at $100{ }^{\circ} \mathrm{C}$ for $12 \mathrm{~h}$ gave none of the corresponding diarylamine 1a (entry 1). We speculated that this disappointing result would arise from sluggish transmetalation. ${ }^{[15]}$ We therefore screened stronger bases in order to accelerate the transmetalation step (entries 1-7). Although $\mathrm{KOH}$ and $\mathrm{KOtBu}$ were not effective, employment of KHMDS provided 1a in 76\% yield (entries 2-4). The choice of a counter cation was important: NaHMDS and LiHMDS only afforded $38 \%$ and $5 \%$ yields of 1a, respectively (entries 5 and 6). The reaction did not proceed in the absence of a palladium catalyst, thereby eliminating a possible $\mathrm{S}_{N} \mathrm{Ar}$ pathway (entry 7). ${ }^{[12]}$ Palladium precursors also affected the efficiency (entry 8-13). SingaCycle-A3, ${ }^{[16]}$ which was commercially available from TCI, showed the higher catalytic activity than Pd-PEPPSI-IPr and 1a was obtained in $84 \%$ yield. Further optimization revealed that the reaction gave the highest yield of $91 \%$ in the presence of 2.5 mol\% of SingaCycle-A3, 1.2 equiv of $p$-toluidine, and 2.5 equiv of KHMDS under concentrated conditions (0.5 M) (entry 13). The choice of the Pd/IPr combination was crucial. Employment of the less bulky IMes ligand or the more bulky IPent ligand showed lower catalytic activity (entry 14 and 15). The reaction with SIPr ligand, which is an analog of IPr with a saturated imidazolium core, also resulted in low yield (entry 16). A combination of Ni salt and IPr ligand was not effective (entry 17).
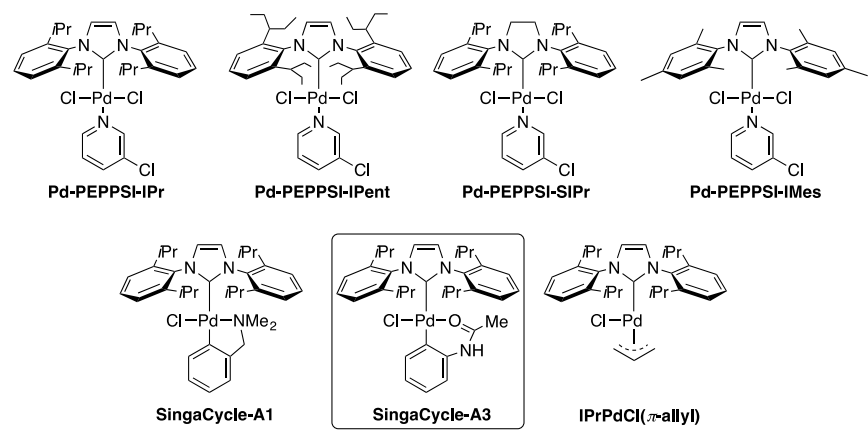

Figure 1. Palladium NHC catalysts used in this paper. 
Table 1. Optimization of the reaction.
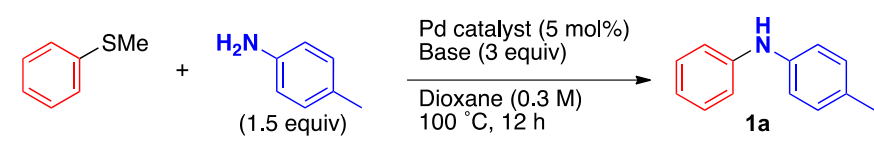

\begin{tabular}{|c|c|c|c|}
\hline Entry & Catalyst & Base & Yield $^{[a]}$ \\
\hline 1 & Pd-PEPPSI-IPr & $\mathrm{K}_{2} \mathrm{CO}_{3}$ & $0 \%$ \\
\hline 2 & Pd-PEPPSI-IPr & $\mathrm{KOH}$ & $0 \%$ \\
\hline 3 & Pd-PEPPSI-IPr & $\mathrm{KOtBu}$ & $7 \%$ \\
\hline 4 & Pd-PEPPSI-IPr & KHMDS/Toluene & $76 \%\left[{ }^{[b]}\right.$ \\
\hline 5 & Pd-PEPPSI-IPr & NaHMDS/THF & $38 \%$ \\
\hline 6 & Pd-PEPPSI-IPr & LiHMDS/THF & $5 \%$ \\
\hline 7 & None & KHMDS/Toluene & $0 \%$ \\
\hline 8 & SingaCycle-A1 & KHMDS/Toluene & $84 \%$ \\
\hline 9 & SingaCycle-A3 & KHMDS/Toluene & $88 \%\left[{ }^{[b]}\right.$ \\
\hline 10 & {$[\mathrm{IPrPdCl}(\pi$-allyl)] } & KHMDS/Toluene & $72 \%$ \\
\hline 11 & $\mathrm{Pd}_{2}(\mathrm{dba})_{3} / \mathrm{IPr} \bullet \mathrm{HCl}$ & KHMDS/Toluene & $50 \%$ \\
\hline 12 & {$\left[(\pi \text {-allyl)PdCl }]_{2} / \mathrm{IPr} \cdot \mathrm{HCl}\right.$} & KHMDS/Toluene & $84 \%$ \\
\hline 13 & SingaCycle-A3 & KHMDS/Toluene & $91 \%[b, c]$ \\
\hline 14 & Pd-PEPPSI-IMes & KHMDS/Toluene & $0 \%$ \\
\hline 15 & Pd-PEPPSI-IPent & KHMDS/Toluene & $5 \%$ \\
\hline 16 & Pd-PEPPSI-SIPr & KHMDS/Toluene & $25 \%$ \\
\hline 17 & $\mathrm{NiBr}_{2} \bullet$ diglyme/IPr•HCl & KHMDS/Toluene & $0 \%$ \\
\hline
\end{tabular}

[a] ${ }^{1} \mathrm{H}$ NMR yield. [b] Isolated yield. [c] $p$-Toluidine (1.2 equiv), SingaCycle-A3 (2.5 mol\%), KHMDS/toluene (2.5 equiv), dioxane (0.5 M).

The scope of this amination was then investigated (Table 2). We first studied the effect of alkylthio leaving groups. Not only odorless 1-dodecylthio group ${ }^{[17]}$ but also bulky $t$-butylthio and phenylthio groups participated in the reaction (entries 1-4). The scope of anilines was also reasonably wide. Bulky o-toluidine reacted smoothly to give $\mathbf{1 c}$ in high yield (entry 6). Anilines having an electron-donating or -withdrawing group engaged in the amination (entries 7-9). Amination with 2-pyridylamine proceeded albeit with a longer reaction time (entry 10). A variety of aryl sulfides were also applicable to the reaction (entries 11-23). Aryl sulfides having various substituents such as o-methyl (entry 13), electron-withdrawing fluoro and trifluoromethyl (entries 14 and 16), electron-donating methoxy (entry 17), protected hydroxy (entry 18), and protected formyl (entry 19) underwent the amination. Unfortunately, 4-trifluoromethylaniline derivative $2 \mathbf{e}$ was not obtained due to the instability of $\mathbf{2 e}$ under the basic conditions. Gratifyingly, the reaction conditions were applicable to bulky polyaromatic sulfides such as 1-naphthyl, 9-phenanthryl, and 9anthracenyl sulfides (entries 20-22). 2-Biphenylylamine 2m was obtained in good yield, which was easily transformed into the corresponding carbazole (vide infra, Table 3).

Since the amination provided potassium diarylamides such as $\mathbf{3}$ before aqueous work-up, one-pot synthesis of triarylamine $\mathbf{4}$ was accomplished by the subsequent reaction of $\mathbf{3}$ with 1bromonaphthalene (eq. 1). ${ }^{[18]}$
Table 2. Scope of the reaction

\begin{tabular}{|c|c|c|c|c|c|}
\hline & & $\begin{array}{l}\text { Sing } \\
\text { KHN }\end{array}$ & $\begin{array}{l}\text { cle-A3 (2.5 n } \\
\text { Toluene ( } 3 \text { e }\end{array}$ & & $\mathrm{H}$ \\
\hline 1 & $\mathrm{Ph}$ & $\mathrm{Me}$ & 4-Me & 1a & $91 \%$ \\
\hline 2 & $\mathrm{Ph}$ & $\mathrm{C}_{12} \mathrm{H}_{25}$ & 4-Me & $1 a$ & $(73 \%)$ \\
\hline 3 & $\mathrm{Ph}$ & $t \mathrm{Bu}$ & 4-Me & $1 a$ & $(65 \%)$ \\
\hline 4 & $\mathrm{Ph}$ & $\mathrm{Ph}$ & 4-Me & $1 a$ & $(99 \%)$ \\
\hline 5 & $\mathrm{Ph}$ & $\mathrm{Me}$ & 3-Me & 1b & $92 \%\left[0^{[b]}\right.$ \\
\hline 6 & $\mathrm{Ph}$ & $\mathrm{Me}$ & 2-Me & 1c & $75 \%$ \\
\hline 7 & $\mathrm{Ph}$ & $\mathrm{Me}$ & 4-MeO & 1d & $90 \%$ \\
\hline 8 & $\mathrm{Ph}$ & $\mathrm{Me}$ & $4-F$ & $1 e$ & $88 \%$ \\
\hline 9 & $\mathrm{Ph}$ & $\mathrm{Me}$ & $3-\mathrm{CF}_{3}$ & $1 f$ & $65 \%{ }^{[b]}$ \\
\hline 10 & $\mathrm{Ph}$ & $\mathrm{Me}$ & (2-Pyridyl) & $1 \mathrm{~g}$ & $75 \%[0, d]$ \\
\hline 11 & 4- $\mathrm{MeC}_{6} \mathrm{H}_{4}$ & Me & 4-Me & $2 a$ & $72 \%{ }^{[c]}$ \\
\hline 12 & 3- $\mathrm{MeC}_{6} \mathrm{H}_{4}$ & Me & 4-Me & $2 b$ & $80 \%{ }^{[c]}$ \\
\hline 13 & $2-\mathrm{MeC}_{6} \mathrm{H}_{4}$ & $\mathrm{Me}$ & 4-Me & 2c & $64 \%$ \\
\hline 14 & $4-\mathrm{FC}_{6} \mathrm{H}_{4}$ & $\mathrm{Me}$ & 4-Me & $2 d$ & $65 \%{ }^{[c]}$ \\
\hline 15 & $4-\mathrm{CF}_{3} \mathrm{C}_{6} \mathrm{H}_{4}$ & $\mathrm{C}_{12} \mathrm{H}_{25}$ & 4-Me & $2 e$ & $0 \%$ \\
\hline 16 & $3-\mathrm{CF}_{3} \mathrm{C}_{6} \mathrm{H}_{4}$ & $\mathrm{C}_{12} \mathrm{H}_{25}$ & 4-Me & $2 f$ & $83 \%$ \\
\hline 17 & $4-\mathrm{MeOC}_{6} \mathrm{H}_{4}$ & $\mathrm{Me}$ & 4-Me & $2 g$ & $73 \%{ }^{[\mathrm{e}]}$ \\
\hline 18 & 4-THPOC $6{ }_{6} \mathrm{H}_{4}$ & $\mathrm{Me}$ & 4-Me & $2 \mathrm{~h}$ & $60 \%{ }^{[\mathrm{b}, \mathrm{d}]}$ \\
\hline 19 & & $\mathrm{Me}$ & 4-Me & $2 \mathrm{i}$ & $76 \%{ }^{[\mathrm{b}]}$ \\
\hline 20 & & $\mathrm{Me}$ & 4-Me & $2 \mathrm{j}$ & $68 \%{ }^{[\mathrm{b}]}$ \\
\hline 21 & & $\mathrm{C}_{12} \mathrm{H}_{25}$ & 4-Me & $2 k$ & $80 \%[f]$ \\
\hline 22 & & $\mathrm{C}_{12} \mathrm{H}_{25}$ & 4-Me & 2I & $81 \%\left[{ }^{[b]}\right.$ \\
\hline 23 & & $\mathrm{Me}$ & 4-Me & $2 m$ & $73 \% \%^{[\mathrm{b}]}$ \\
\hline
\end{tabular}

[a] Isolated yield. ${ }^{1} \mathrm{H}$ NMR yields are in the parentheses. [b] SingaCycle-A3 (10 mol\%). [c] SingaCycle-A3 (5 mol\%). [d] $18 \mathrm{~h}$. [e] SingaCycle-A3 (2.5 mol\% x 3). [f] SingaCycle-A3 (7.5 mol\%).

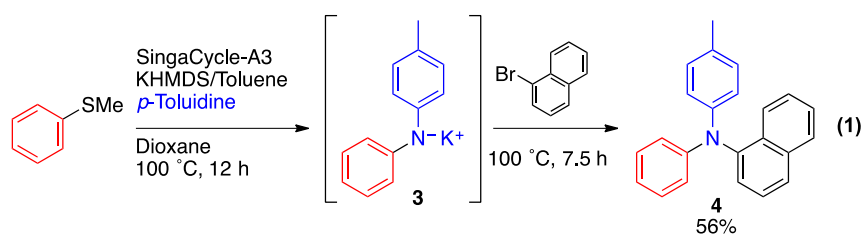

Taking advantage of the efficient amination of bulky aryl sulfides (e.g., Table 2, entry 23), we tried to achieve modular synthesis of carbazoles. Compounds 6 were obtained through palladium-catalyzed Suzuki reaction of 2-bromoaryl methyl sulfide $\mathbf{5 a}$ or $\mathbf{5 b}$, which are readily available through bromination of aryl methyl sulfide. The amination of $\mathbf{6}$ provided the corresponding amine 7. Although a variety of direct oxidative $\mathrm{C}-\mathrm{N}$ bond formation to construct carbazoles have been reported, ${ }^{[19,20]}$ the substituents on nitrogen were limited to alkyl, ${ }^{[20 a]}$ acyl, ${ }^{[20 b-d]}$ sulfony ${ }^{[20 \mathrm{e}-\mathrm{g}]}$ or pyridy ${ }^{[20 h, i]}$ groups. We therefore developed new conditions for $N$-aryl substrates 7. After extensive screening, we found $\mathrm{Cu}(\mathrm{OAc})_{2}$ was the best oxidant to furnish carbazole 8 in high 
Angewandte Communications

yield (See SI for optimization). A wide variety of carbazoles including a ladder-type molecule $\mathbf{8 e}$, were obtained through this three-step strategy in $24-40 \%$ overall yield from 5 . Interestingly, the ring-closing reaction of $\mathbf{7 d}$ proceeded regioselectively at the ortho position of the methoxy group to give $\mathbf{8 d}$.

Table 3. Modular synthesis of $N$-arylcarbazoles.

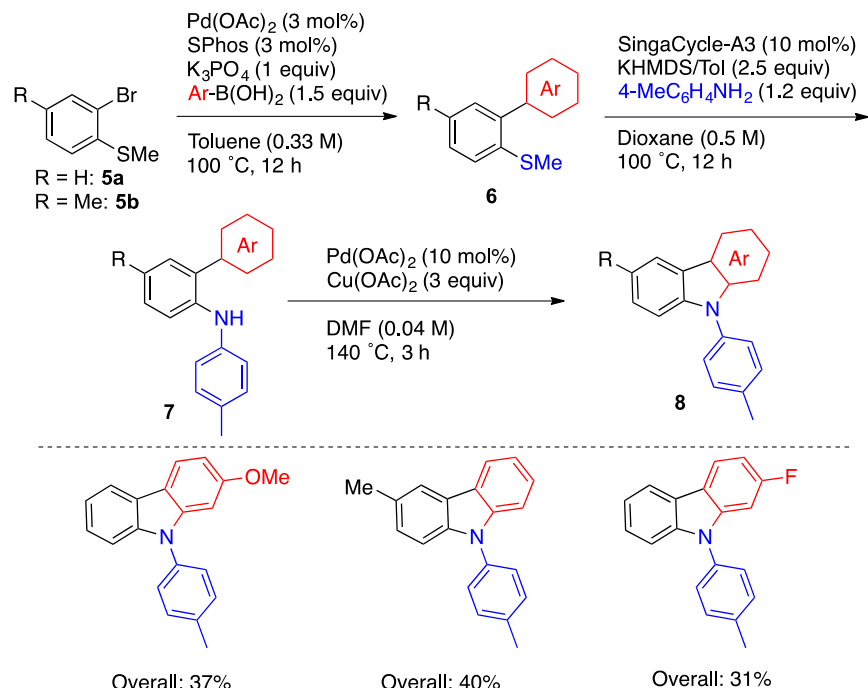

(6a:76\%, 7a:77\%, 8a:63\%) (6b:84\%, 7b:75\%, 8b:64\%)(6c:76\%, 7c:60\%[a], 8c:68\%[b])
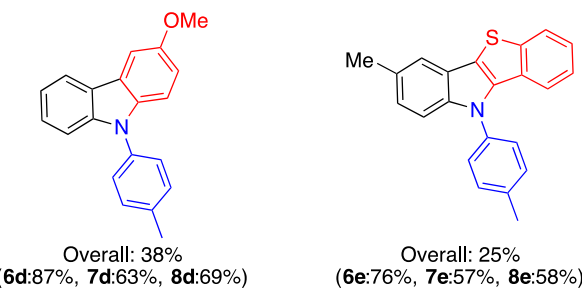

[a] 2 h. [b] $150{ }^{\circ} \mathrm{C}$

We have regarded sulfur as a halogen surrogate of higher functionality because of the recent drastic boost in extended Pummerer reactions ${ }^{[14,21-23]}$ where sulfoxide moieties are converted to the corresponding sulfides along with intriguing $\mathrm{C}-\mathrm{C}$ bond formation. Since the limited variety of transformations of the resulting alkylthio moieties are available, ${ }^{[24]}$ we have been striving after new repertoires for transformations of $\mathrm{C}-\mathrm{S}$ bonds to expand the potential of the Pummerer products. ${ }^{[14]}$ Naturally, we envisioned that a combination of extended Pummerer reactions with our catalytic amination would provide a new tool for synthesizing nitrogen-containing aromatic compounds. For example, we indeed employed our benzothiophene synthesis from readily available ketene dithioacetal monoxide $\mathbf{9}^{[22 \mathrm{~b}]}$ (Scheme 1). The Pummerer cyclization of $\mathbf{9}$ with $\mathrm{Tf}_{2} \mathrm{O}$ followed by demethylation with ethanolamine afforded 2-methylthio-3-phenylbenzothiophene (11) in $90 \%$ yield. The resulting methylthio group of $\mathbf{1 1}$ was replaced with $p$-toluidine through our technology. Another example includes Procter's ortho-propargylation of aryl sulfoxides, which afforded methyl 2-propargylphenyl sulfide 15. ${ }^{[23 b]}$ Exposure of $\mathbf{1 5}$ under the amination conditions beneficially led to tandem amination/annulation to yield the corresponding indole $\mathbf{1 6}$ in $81 \%$ yield, probably via possible intermediate 17. ${ }^{[25]}$
In summary, we have developed new amination of aryl sulfides by using a state-of-the-art Pd/IPr catalyst. The scope is wide enough to employ various aryl sulfides and anilines including bulky orthosubstituted substrates. Amination of 2-biphenylyl sulfides followed by intramolecular $\mathrm{C}-\mathrm{N}$ bond formation under our modified oxidative conditions has opened a door to modular synthesis of $\mathrm{N}$ arylcarbazoles. We have also demonstrated that extended Pummerer reaction/amination sequences are highly efficient for rapid synthesis of complex molecules. Our work will be an important milestone to trigger a paradigm shift from $\mathrm{C}-\mathrm{X}$-based synthesis to C-S-based synthesis.
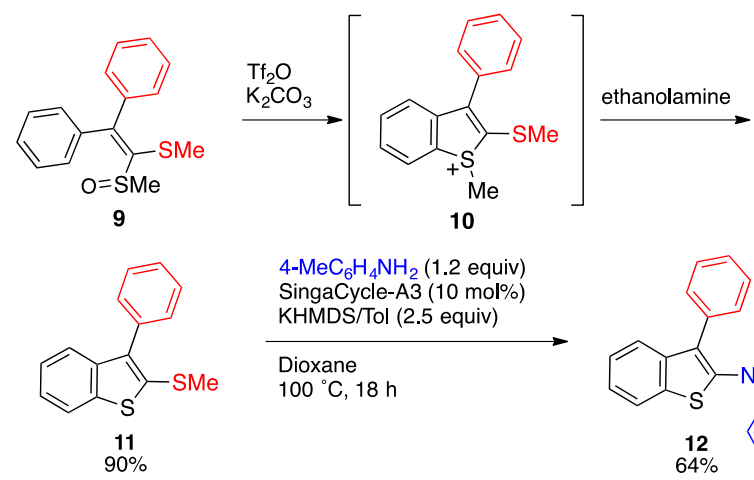

4- $\mathrm{MeC}_{6} \mathrm{H}_{4} \mathrm{NH}_{2}$ (1.2 equiv) SingaCycle-A3 (10 mol\%) $\mathrm{KHMDS} / \mathrm{Tol}$ (2.5 equiv)

Dioxane

$100^{\circ} \mathrm{C}, 18 \mathrm{~h}$

$90 \%$
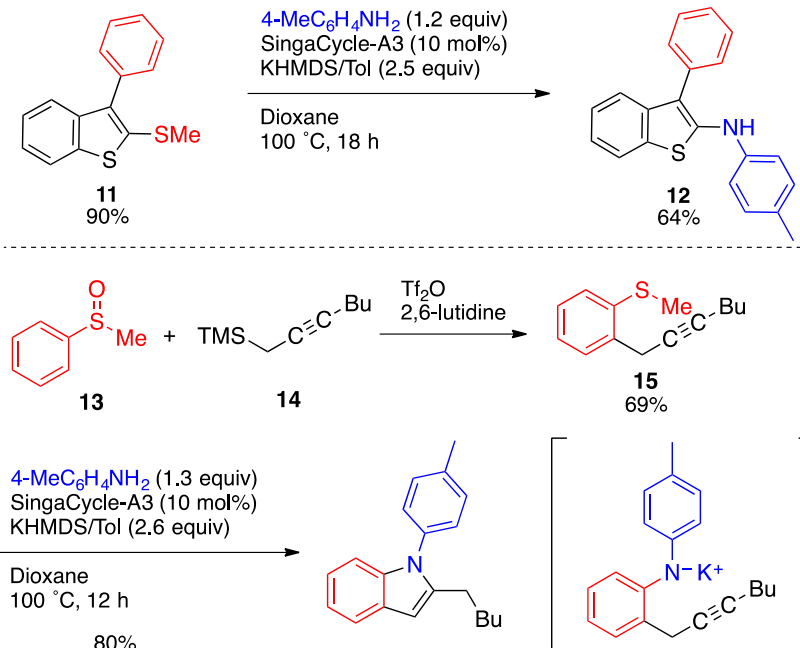

$80 \%$
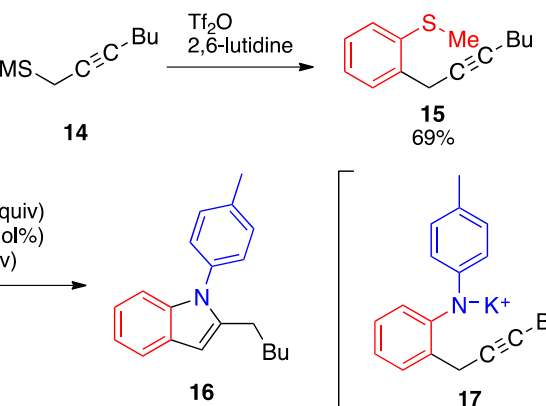

15

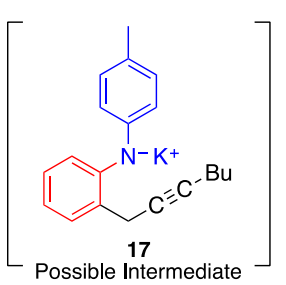

Scheme 1. Extended Pummerer/amination sequences.

\section{Experimental Section}

Synthesis of $\mathbf{1 a}$ is representative (Table 1, entry 13). p-Toluidine (64 mg, 0.6 $\mathrm{mmol})$ and SingaCycle-A3 $(8.3 \mathrm{mg}, 0.013 \mathrm{mmol})$ were added to a Schlenk tube under argon. Dioxane $(1 \mathrm{~mL})$ and thioanisole $(59 \mu \mathrm{L}, 0.50 \mathrm{mmol})$ were added successively to the tube. After an addition of KHMDS $(2.5 \mathrm{~mL}, 1.25 \mathrm{mmol}, 0.5$ $\mathrm{M}$ in toluene), the mixture was heated at $100{ }^{\circ} \mathrm{C}$ for $12 \mathrm{~h}$. After the reaction, the mixture was cooled to $25{ }^{\circ} \mathrm{C}$. The reaction was then quenched with water (5 $\mathrm{mL})$. The organic compounds were extracted with EtOAc $(5 \mathrm{~mL})$ three times. The combined organic part was then washed with brine. The mixture was filtered through a pad of silica-gel and $\mathrm{Na}_{2} \mathrm{SO}_{4}$ and concentrated in vacuo. Purification by chromatography on silica-gel $(n$-hexane/EtOAc $=20 / 1)$ provided 1 a (83 mg, $0.45 \mathrm{mmol}, 91 \%)$.

Received: ((will be filled in by the editorial staff))

Published online on ((will be filled in by the editorial staff))

Keywords: aryl sulfide $\bullet$ palladium $\bullet$ amination $\bullet$ extended Pummerer reaction

[1] Recent reviews: a) D. S. Surry, S. L. Buchwald, Angew. Chem. 2008, 120, 6438; Angew. Chem. Int. Ed. 2008, 47, 6338; b) J. F. Hartwig, Acc. Chem. Res. 2008, 41, 1534; c) T. R. M. Rauws, B. U. W. Maes, Chem. Soc. Rev. 2012, 41, 2463; d) J. Magano, J. R. Dunetz, Chem. 
Angewandte Communications

Rev. 2011, 111, 2177; e) C. Torborg, M. Beller, Adv. Synth. Catal. 2009, 351, 3027; f) C. Valente, S. Çalimsiz, K. H. Hoi, D. Mallik, M. Sayah, M. G. Organ, Angew. Chem. 2012, 124, 3370; Angew. Chem. Int. Ed. 2012, 51, 3314; g) I. P. Beletskaya, A. V. Cheprakov, Organometallics 2012, 31, 7753; h) E. A. B. Kantchev, C. J. O’Brien, M. G. Organ, Aldrichim. Acta 2006, 39, 97; i) G. C. Fortman, S. P Nolan, Chem. Soc. Rev. 2011, 40, 5151. Recent books: j) J. Hartwig, Organotransition Metal Chemistry, University Science Books, Mil Valley, 2010; k) A. de Meijere, F. Diederich, Metal-Catalyzed CrossCoupling Reactions, 2nd ed., Wiley-VCH, Weinheim, 2004; l) Handbook of Organopalladium Chemistry for Organic Synthesis (Ed. E.-i. Negishi), Wiley, New York, 2002; m) Applied Cross-Coupling Reactions (Ed.: Y. Nishihara), Springer, 2013.

[2] Very recent selected examples: a) Y. Suzuki, N. Fukui, K. Murakami, H. Yorimitsu, A. Osuka, Asian J. Org. Chem. 2013, 2, 1066; b) S. Ge, R. A. Green, J. F. Hartwig, J. Am. Chem. Soc. 2014, 136, 1617; c) M. Su, N. Hoshiya, S. L. Buchwald, Org. Lett. 2014, 16, 832; d) T. Hatakeyama, R. Imayoshi, Y. Yoshimoto, S. K. Ghorai, M. Jin, H. Takaya, K. Norisuye, Y. Sohrin, M. Nakamura, J. Am. Chem. Soc. 2012, 134, 20262.

[3] a) L.-L. Li, E. W.-G. Diau, Chem. Soc. Rev. 2013, 42, 291; b) K Walzer, B. Maennig, M. Pfeiffer, K. Leo, Chem. Rev. 2007, 107, 1233; c) Y. Shirota, H. Kageyama, Chem. Rev. 2007, 107, 953.

[4] J. A. Bikker, N. Brooijmans, A. Wissner, T. S. Mansour, J. Med. Chem. 2009, 52, 1493.

[5] a) B. P. Fors, D. A. Watson, M. R. Biscoe, S. L. Buchwald, J. Am. Chem. Soc. 2008, 130, 13552; b) C. M. So, Z. Zhou, C. P. Lau, F. Y. Kwong, Angew. Chem. 2008, 120, 6502; Angew. Chem. Int. Ed. 2008, 47, 6402.

[6] a) B. C. Hamann, J. F. Hartwig, J. Am. Chem. Soc. 1998, 120, 7369; b) A. H. Roy, J. F. Hartwig, J. Am. Chem. Soc. 2003, 125, 8704; c) T. Ogata, J. F. Hartwig, J. Am. Chem. Soc. 2008, 130, 13848; d) C.-Y. Gao, L.-M. Yang, J. Org. Chem. 2008, 73, 1624.

[7] a) L. Hie, S. D. Ramgren, T. Mesganaw, N. K. Garg, Org. Lett. 2012, 14, 4182; b) T. Mesganaw, A. L. Silberstein, S. D. Ramgren, N. F. F. Nathel, X. Hong, P. Liu, N. K. Garg, Chem. Sci. 2011, 2, 1766.

[8] a) S. D. Ramgren, A. L. Silberstein, Y. Yang, N. K. Garg, Angew. Chem. 2011, 123, 2219; Angew. Chem. Int. Ed. 2011, 50, 2171; b) H. Tadaoka, T. Yamakawa, Tetrahedron Lett. 2012, 53, 5531; c) L. Ackermann, R. Sandmann, W. Song, Org. Lett. 2011, 13, 1784.

[9] T. Shimasaki, M. Tobisu, N. Chatani, Angew. Chem. 2010, 122, 2991; Angew. Chem. Int. Ed. 2010, 49, 2929.

[10] J.-H. Huang, L.-M. Yang, Org. Lett. 2011, 13, 3750.

[11] Nickel-catalyzed amination of aryl methyl ethers of 2methoxynaphthalene or arenes having an electron-withdrawing group: M. Tobisu, T. Shimasaki, N. Chatani, Chem. Lett. 2009, 38, 710.

[12] Examples of $S_{N} A r$ reactions of electron-deficient aryl sulfides: a) R. Chamberlin, M. R. Crampton, J. Chem. Soc., Perkin Trans. 21993 75; b) Y. Dong, B. Liu, P. Chen, Q. Liu, M. Wang, Angew. Chem. 2014, 126, 3510; Angew. Chem. Int. Ed. 2014, 53, 3442. Aryl sulfone: c) W. ten Hoeve, C. G. Kruse, J. M. Luteyn, J. R. G. Thiecke, H. Wynberg, J. Org. Chem. 1993, 58, 5101. Aryl sulfoxides: d) J. L. G. Ruano, A. Parra, V. Marcos, C. del Pozo, S. Catalán, S. Monteagudo, S. Fustero, A. Poveda J. Am. Chem. Soc. 2009, 131, 9432.

[13] Smiles rearrangement of 4-nitrothiophenol to 4-nitroaniline: $M$. Mizuno, M. Yamano, Org. Lett. 2005, 7, 3629.

[14] a) Y. Ookubo, A. Wakamiya, H. Yorimitsu, A. Osuka, Chem. Eur. J. 2012, 18, 12690; b) K. Murakami, H. Yorimitsu, A. Osuka, Angew. Chem. Int. Ed. 2014, 53, in press, DOI: 10.1002/anie.201403288.
[15] In order to enhance the rate of the transmetalation step, CuTC was employed in the reactions of arylboronic acids with thioesters: L. S. Liebeskind, J. Srogl, J. Am. Chem. Soc. 2000, 122, 11260. Unfortunately, the addition of CuTC was not effective to this amination reaction.

[16] E. A. B. Kantchev, J. Y. Ying, Organometallics 2009, 28, 289

[17] M. Node, K. Kumar, K. Nishide, S. Ohsugi, T. Miyamoto, Tetrahedron Lett. 2001, 42, 9207.

[18] Reviews on the importance of one-pot multistep syntheses: a) S. Suga, D. Yamada, J. Yoshida, Chem. Lett. 2010, 39, 404 and references cited therein; b) A. J. Bard, Integrated Chemical Systems, Wiley, New York, 1994; c) Multicomponent Reactions (Eds: J. Zhu, H. Bienaymé), Wiley, Weinheim, 2005.

[19] Recent review: a) M.-L.Louillat, F. W. Patureau, Chem. Soc. Rev. 2014, 43, 901; b) N. Yoshikai, Y. Wei, Asian J. Org. Chem. 2013, 2, 466; c) T. Jin, J. Zhao, N. Asao, Y. Yamamoto, Chem. Eur. J. 2014, 20, 3554.

[20] Recent examples: a) J. A. Jordan-Hore, C. C. C. Johansson, M. Gulias, E. M. Beck, M. J. Gaunt, J. Am. Chem. Soc. 2008, 130, 16184; b) W. C. P. Tsang, N. Zheng, S. L. Buchwald, J. Am. Chem. Soc. 2005, 127, 14560; c) B.-J. Li, S.-L. Tian, Z. Fang, Z.-J. Shi, Angew. Chem. 2008, 120, 1131; Angew. Chem. Int. Ed. 2008, 47, 1115; d) W. C. P. Tsang, R. H. Munday, G. Brasche, N. Zheng, S. L. Buchwald, J. Org. Chem. 2008, 73, 7603; e) S. W. Youn, J. H. Bihn, B. S. Kim, Org. Lett. 2011, 13, 3738; f) K. Inamoto, T. Saito, K. Hiroya, T. Doi, Synlett 2008, 3157; g) S. H. Cho, J. Yoon, S. Chang, J. Am. Chem. Soc. 2011, 133, 5996; h) J.-H. Chu, P.-S. Lin, Y.-M. Lee, W.-T. Shen, M.-J. Wu, Chem. Eur. J. 2011, 17, 13613; i) J.-H. Chu, H.-P. Huang, W.-T. Hsu, S.-T. Chen, M.-J. Wu, Organometallics 2014, 33, 1190.

[21] Reviews : a) L. H. S. Smith, S. C. Coote, H. F. Sneddon, D. J. Procter, Angew. Chem. 2010, 122, 5968; Angew. Chem., Int. Ed. 2010, 49, 5832; b) S. Akai, Y. Kita, Top. Curr. Chem. 2007, 274, 35; c) K. S. Feldman, Tetrahedron 2006, 62, 5003; d) S. K. Bur, A. Padwa, Chem. Rev. 2004, 104, 2401; e) Sulfur-Mediated Rearrangements I (Ed.: E. Schaumann), Springer, 2007.

[22] From our group: a) H. Yorimitsu, J. Synth. Org. Chem., Jpn. 2013, 71, 341; b) S. Yoshida, H. Yorimitsu, K. Oshima, Org. Lett. 2007, 9, 5573; c) S. Yoshida, H. Yorimitsu, K. Oshima, Org. Lett. 2009, 11, 2185; d) T. Kobatake, S. Yoshida, H. Yorimitsu, K. Oshima, K. Angew. Chem. 2010, 122, 2390; Angew. Chem., Int. Ed. 2010, 49, 2340; e) K. Murakami, J. Imoto, H. Matsubara, S. Yoshida, H. Yorimitsu, K. Oshima, Chem. Eur. J. 2013, 19, 5625; f) S. Yoshida, H. Yorimitsu, K. Oshima, Chem. Lett. 2008, 37, 786; g) T. Kobatake, D. Fujino, S. Yoshida, H. Yorimitsu, K. Oshima, J. Am. Chem. Soc. 2010 132, 11838.

[23] Recent examples from other groups: a) X. Huang, M. Patil, C. Farès, W. Thiel, N. Maulide, J. Am. Chem. Soc. 2013, 135, 7312; b) A. J. Eberhart, D. J. Procter, Angew. Chem. 2013, 125, 4100; Angew. Chem., Int. Ed. 2013, 52, 4008; c) K. S. Feldman, M. D. Fodor, J. Org. Chem. 2009, 74, 3449; d) S. Akai, N. Kawashita, Y. Wada, H. Satoh, A. H. Alinejad, K. Kakiguchi, I. Kuriwaki, Y. Kita, Tetrahedron Lett. 2006, 47, 1881.

[24] Reviews of transformations of alkylthio groups: a) S. R. Dubbaka, P. Vogel, Angew. Chem. 2005, 117, 7848; Angew. Chem., Int. Ed. 2005, 44, 7674; b) T.-Y. Luh, Z.-J. Ni, Synthesis 1990, 89; c) T. Y. Luh, Acc. Chem. Res. 1991, 24, 257; d) F. Pan, Z.-J. Shi, ACS Catal. 2014, 4, 280.

[25] a) B. Das, P. Kundu C. Chowdhury, Org. Biomol. Chem. 2014, 12, 741; b) A. Varela-Fernández, J. A. Varela, C. Saá, Adv. Synth. Catal. 2011, 353, 1933. 


\section{Angewandte \\ Communications}

\section{Synthetic Method}

Tomohiro Sugahara, Kei Murakami, * Hideki Yorimitsu, ${ }^{*}$ and Atsuhiro Osuka Page - Page

Palladium-Catalyzed Amination of Aryl Sulfides with Anilines

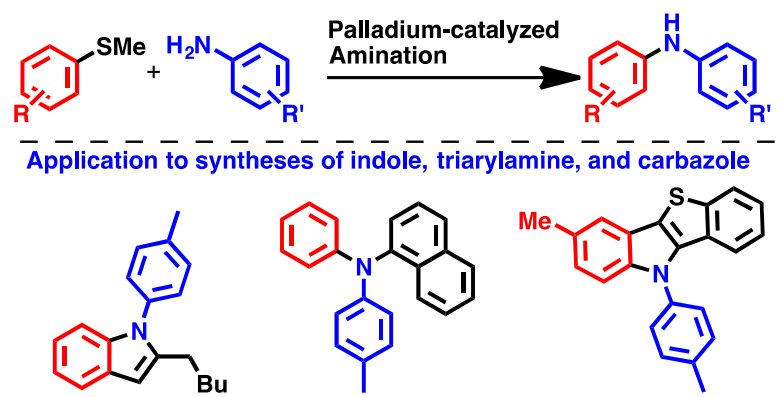

Transformation of inert $\mathrm{C}-\mathrm{S}$ bonds of aryl sulfides into $\mathrm{C}-\mathrm{N}$ bonds has been accomplished by using a palladium complex having an $\mathrm{N}$-heterocyclic carbene ligand. A wide range of aryl sulfides and anilines are applicable to the reaction. Thanks to the efficient conditions for amination of bulky sulfides, modular syntheses of carbazoles from 2-biphenylylamine products were achieved through our newly modified oxidative $\mathrm{C}-\mathrm{N}$ bond formation. When combined with extended Pummerer reactions that afforded useful aryl sulfides, the amination led to highly efficient construction of intriguing nitrogen-containing molecules. 